\title{
Correction to: A failure case study of tunnel water inrush source identified by statistical analysis model
}

\author{
Li-Gang Yuan $^{1,2} \cdot$ Xiao-Li Li $^{1} \cdot$ Xin $\mathrm{Li}^{1} \cdot$ Yi-Lin Yu ${ }^{1} \cdot$ Li-Guang Chen $^{1} \cdot$ Xian-Pan Xu ${ }^{1,2} \cdot$ Xiang Wang $^{1}$ \\ Published online: 7 October 2021 \\ (c) Saudi Society for Geosciences 2021
}

Correction to: Arabian Journal of Geosciences (2021) 14:1809

https://doi.org/10.1007/s12517-021-08200-1

In this article the author name "Li-guang Chen" was incor-

rectly written as "Li-gang Chen".

The original article can be found online at https://doi.org/10.1007/

s12517-021-08200-1.

Yi-Lin Yu

tunnelHWZ@csu.edu.cn

1 China Construction Municipal Engineering Corporation

Limited, Beijing 100000, China

2 China Construction First Group Corporation Limited, Beijing 100000, China 